\title{
Progressive Resistive Exercise in Weaning High Quadriplegics from the Ventilator
}

\author{
R. M. Lerman, M.D., ${ }^{1}$ M. S. Weiss, M.D. ${ }^{2}$ \\ ${ }^{1}$ Resident, Physical Medicine and Rehabilitation, Stanford University Affiliated \\ Hospitals, 1227 Windsor Way, Redwood City, California 19160. ${ }^{2}$ Assistant Clinical \\ Professor, Stanford University Medical Center, Family, Community and Preventive \\ Medicine, Health Research and Policy Building Room 106A, Stanford, California \\ 94305, U.S.A.
}

\begin{abstract}
Summary
Acutely high level quadriplegics may experience neuromuscular respiratory insufficiency secondary to loss of use of intercostal and abdominal muscles as well as partial involvement of the phrenic nerve. Frequently, these patients will require mechanical ventilation in the initial stages of their treatment. These patients may present difficulty with weaning off the ventilator. In addition, poor respiratory reserve increases the risk of episodic decompensation. We have instituted a progressive resistive exercise protocol (PRE) analogous to $P R E$ commonly used in training skeletal muscle, to wean patients off the ventilator. This involves determining the patient's endurance to the development of fatigue while off the ventilator. Patients are re-evaluated weekly until they are weaned from the ventilator. Three case studies are reported in which this protocol was used. In addition to our standard respiratory therapy and physical therapy protocols, values for vital capacity and maximum inspiratory force at admission and post-weaning were recorded. After completion of the programme, none of the patients required re-intubation or subsequent mechanical ventilation. This method of diaphragm training may be useful in weaning high level quadriplegics from the ventilator.
\end{abstract}

\section{Introduction}

Respiratory complications are a major source of morbidity and mortality in the acute quadriplegic population, particularly high level quadriplegics (McMichan, 1980). The majority of patients with intact diaphragm function (normal neurological function through C5) can be managed without mechanical ventilation or can be quickly weaned from the ventilator provided there is no premorbid history of respiratory disease. However, mechanical ventilation is often required secondary to loss of control of muscles used in respiration including abdominal muscles, intercostal muscles and in some cases partial or total loss of diaphragmatic function.

The diaphragm is the major muscle of respiration. Shaffer estimates that it is 
responsible for $2 / 3$ of respiration while a patient is sitting and $3 / 4$ while the patient is supine (Shaffer, 1981). Intercostal muscles relieve the diaphragm in times of fatigue and aid with expansion of the rib cage. Accessory muscles of respiration, the scaleni, sternomastoids and the abdominal muscles, play a minimal role in normal respiration. The abdominal muscles aid forced expiration as well (Haas, 1979).

We looked at a subset population of patients with partial diaphragmatic control, with C3/4 complete lesions. Past experience had shown that this was a more difficult population to wean from mechanical ventilation. We developed an exercise protocol analogous to the principles of progressive resistive exercises to train the partially innervated diaphragm in order to wean these patients from the ventilator. Three patients are presented in which this protocol was used. One of the patient's had been weaned without the protocol, however, re-intubation was required and the patient was subsequently weaned.

\section{Patients}

Patients were examined and the neurological level determined using Frankel classification. Vital capacity (VC) and maximum inspiratory force (MIF) were measured and recorded at the time patients were entered into the study and when they were weaned from the ventilator. These measurements were also taken throughout the hospital course. VC was measured and recorded at discharge. Patients were evaluated off the ventilator to determine endurance to the development of fatigue (fatigue determined by a $20^{\circ}$ o decrement of $\mathrm{VC}$ or MIF). This time was recorded and the patient was taken off the ventilator and placed on a T-tube for that length of time QID. Patients were closely monitored for respiratory distress by following the vital signs and checking the arterial blood gases. Patients were re-evaluated on a weekly basis and T-tube time was increased accordingly, usually by $15-20^{\circ}$. . As T-tube times became progressively longer, the number of sessions was decreased to TID and then BID, maintaining at least 2 hours recovery period. When the patient was tolerating two 10 hour sessions they were changed to one daily session with maximum T-tube time determined as before. This was subsequently increased until the patient was independent from the ventilator.

In addition, patients were treated with aggressive respiratory therapy (Table I) and enrolled in breathing class held by physiotherapy once they were off the ventilator 1 hour per day. Class is 30 minutes per day and is standard for all patients in the spinal cord injury service (Table II).

\section{Case Report A}

A 22-year-old male involved in a motor vehicle accident on August 11, 1984, in which he sustained a rotatory fracture of $\mathrm{C} 3$ and $\mathrm{C} 4$ with a unilateral locked facet. This resulted in C3/4 incomplete or C3/4 Frankel B quadriplegia. Reduction was achieved with traction. The patient developed pneumonia in the first week after injury, and was successfully treated with antibiotics. The patient was weaned from the ventilator initially with $T$-tube trials 1 hour long every 
Table I Respiratory Therapy Protocol

1. Intermittent Positive Pressure Breathing (IPPB) every 4 to 6 hours using bronchodilators and/or mucolytics as indicated.

2. Patients are placed in the Trendelenberg position for 20 minutes each morning for postural drainage with chest percussion, vibration, manual assisted coughing and suctioning. This is followed until vital capacity reaches greater than 2000 .

3. Postural drainage and percussion with manual assisted coughing and suctioning every 4 to 6 hours (in addition to above) as indicated.

4. Obtain vital capacity measurements on newly admitted quadriplegic patients every shift for 72 hours while awake, then daily for 1 week, then every week for 4 weeks, and then once per month until discharged.

Table II Breathing Class

1. Deep breathing: The patient inhales deeply through his mouth and holds breath 1-2 seconds then exhales slowly through the mouth. Repeat five times.

2. Triplets: The patient takes a deep breath through his mouth, holds it and takes another breath, holds it and takes one more, then exhales. Repeat five times.

3. Quick breaths: The patient inhales through his mouth as much air as he can as quickly as he can using two quick breaths. Repeat ten times.

4. Numbers counting: The patient inhales as deeply as he can, counts out loud until he runs out of air. Repeat two times.

5. Accessory for neck: The patient tightens his neck muscles then relaxes. Two sets of fifteen repetitions.

6. Accessory for upper trapezius: The patient shrugs shoulders then relaxes. Two sets of fifteen repetitions.

7. Coughing: The patient inhales deeply through his mouth then performs two quick coughs. Repeat three times.

other hour while the patient was awake. This was subsequently increased to 2 hours out of every 3. The patient was then allowed to T-tube as tolerated as long as the vital capacity was greater than 1000 .

The ventilator was discontinued on September 6, 1984; however, the patient was found to be retaining $\mathrm{CO}_{2}$ and was placed back on the ventilator on September 12, 1984. A double exposure x-ray revealed a normal left hemidiaphragm with no right hemidiaphragm movement. The patient was initiated on the weaning protocol on September 12, 1984. T-tube tolerance was initially 2 hours per session.

Repeat double exposure chest $\mathrm{x}$-rays revealed minimal right hemidiaphragm movement. The patient improved his vital capacity and the ventilator was discontinued on November 7, 1984.

The patient was noted on November 5, 1984, to have a new trace of left upper extremity movement. This progressed slowly and at the time of discharge, January 8, 1985, there was a trace to poor motor strength in the left upper and lower extremities and a trace distal right upper extremity movement. The patient had no respiratory complications after the ventilator was discontinued. 


\section{Case Report B}

A 47-year-old female involved in a motor vehicle accident on September 4, 1984, sustaining a fracture dislocation of $\mathrm{C} 4 / 5$ which resulted in $\mathrm{C} 4$ complete or C4 Frankel A. There was no volitional movement below C3 with sensory to C4. Patient was admitted to the Santa Clara Valley Medical Center's spinal cord injury service on September 5, 1984, being transferred on a ventilator. Multiple medical problems ensued, including refractory gastrointestinal bleeding felt to be secondary to ulcerated lesions of the colon requiring ileostomy. In addition, a patient with a right lower lobe pneumonia was treated successfully.

On October 3, 1984, she was started on the weaning protocol which was well tolerated. Initial T-tube tolerance was $1 \frac{1}{2}$ hours. On November 9, 1984, ventilator was discontinued. The patient completed her rehabilitation without further respiratory difficulties and was discharged on March 3, 1985.

\section{Case Report C}

A 24-year-old male was well until October 23, 1984, when he was involved in a diving accident in which he sustained a teardrop fracture of $\mathrm{C} 4$ and $\mathrm{C} 5$ with posterior displacement of C5 on C6. This resulted in C4 Frankel A or C4 complete quadriplegia with impaired shoulder protraction and elevation. Sensation was intact to pinprick in $\mathrm{C} 4$ on the left and partial $\mathrm{C} 4$ on the right. The patient was intubated in the emergency room. He developed left lower lobe atelectasis and was treated with antibiotics. He was transferred to Santa Clara Valley Medical Center's spinal cord injury service on November 1, 1984.

On November 7, 1984, double exposure chest $\mathrm{x}$-ray revealed decreased movement of the right hemidiaphragm and no left hemidiaphragm movement. On November 14, 1984, he was started on the weaning protocol. Initial T-tube time was 10 minutes. His improvement progressed and the ventilator was discontinued on January 8, 1985.

His illness was complicated by the development of subglottic stenosis which required to be excised on February 2, 1985. Pseudomonas pneumonia occurred in March, 1985, and was treated with Piperacillin and Tobramycin. Re-intubation was not necessary. The remainder of his hospital course was unremarkable and he was discharged on April 26, 1985.

\section{Results}

All three patients showed increase in their vital capacity and MIF while on the protocol. They continued to make gains in vital capacity after mechanical ventilation was discontinued (Table III). Patients were neurologically stable through the course of the study. No respiratory decompensation occurred necessitating re-intubation. 
Table III After Mechanical Ventilation was Discontinued

\begin{tabular}{|c|c|c|c|c|c|c|c|}
\hline & \multicolumn{2}{|c|}{ Admission } & \multicolumn{2}{|c|}{$\mathrm{D} / \mathrm{C}$ ventilator } & \multirow{2}{*}{$\begin{array}{l}\text { Length of time } \\
\text { to wean/day }\end{array}$} & \multirow{2}{*}{\multicolumn{2}{|c|}{$\begin{array}{c}\text { D/C hospital } \\
\text { VC- }{ }_{0} \text { predicted }\end{array}$}} \\
\hline & MIF & VC & MIF & VC & & & \\
\hline A & -28 & 800 & -40 & 1230 & 58 & 1540 & $28^{\circ}{ }_{0}$ \\
\hline B & -30 & 1250 & -40 & 1250 & 40 & 1570 & $66^{\circ}$ \\
\hline $\mathrm{C}$ & -13 & 300 & -22 & 1100 & 54 & 1150 & $22^{\circ}{ }_{0}^{\circ}$ \\
\hline
\end{tabular}

\section{Discussion}

The diaphragm is a specialised skeletal muscle. It has been shown the diaphragm responds like skeletal muscle in terms of fatiguability and training (Cohen, 1982). Lieberman found that $55^{\circ}$ o of the fibres in the human diaphragm are red, slow twitch fibres. Roussos and Macklen found at less than $40^{\circ} \%$ of maximum generated pressure at FRC, the diaphragm will contract indefinitely without fatigue. At greater than $40^{\circ}$ o the diaphragm will eventually fatigue (Roussos, 1977). Diaphragm fatigue has been determined through EMG studies (Bellemare, 1982).

In normal persons when fatigue occurs, the intercostal muscles and accessory muscles become active. In spinal cord injured patients, the diaphragm is acutely prone to fatigue. The intercostal muscle control is lost and the chest becomes flaccid. With diaphragmetic contraction, the rib cage may be pulled in without stabilisation. The abdominal muscles which function to maintain pressure in the abdomen in order to place the diaphragm at a mechanical advantage are also absent. In addition, there may be decreased lung compliance secondary to infections and/or impaired clearing of secretions due to decreased expiratory mechanism and cough (Gross, 1980).

Quadriplegics will fatigue at resistances of 5 to $17.5 \mathrm{~cm} \mathrm{H}_{2} \mathrm{O} / 1$-sec compared to 70 to $87 \mathrm{~cm} \mathrm{H}_{2} \mathrm{O} / 1$-sec in normals (Gross, 1980). Therefore, the quadriplegic is prone to fatigue and has less ability to compensate resulting in respiratory insufficiency. In patients with partial loss of diaphragm control intubation is almost a certainty. The approach taken in these patients was to train the diaphragm using a strengthening program with the goal to reduce fatigability and allow weaning from the ventilator. Diaphragm training has been demonstrated in normal and diseased patients (Gross, 1980; Fugl-Meyer, 1972; Leith, 1976). Studies have been performed on quadriplegic patients in which strength and endurance have been increased. We specifically studied patients with partial innervation of the diaphragm who were ventilator dependent. Improved strength was measured by MIF and VC, improved endurance marked by successful weaning from the ventilator.

Our protocol uses the principles of progressive resistance exercise, in which the time off the ventilator is increased on a weekly basis to the point of diaphragm fatigue, as weight is increased in an upper extremity strengthening program.

The natural history of quadriplegic patients is improved respiratory status as spinal shock resolves and spasticity of the rib cage stabilises the chest. In addition, resolution of edema and inflammation about the cord may lower the presenting spinal cord level. While these changes may have been a factor in our 
patients, no significant changes in neurological level were noted on physical exam or by double exposure chest x-ray. Patients A and B were placed on the protocol 1 month after injury which was post spinal shock. Patient A was weaned from the ventilator by a different method and decompensated with no evidence of intrinsic lung disease. This was felt to be secondary to poor conditioning. He was subsequently placed on the protocol and did well. The patients presented improved while on the protocol, we feel, secondary to the training effect on the diaphragm.

\section{Conclusion}

Respiratory complications are a major source of morbidity and mortality in the quadriplegic population, particularly acutely. We have instituted a protocol for weaning quadriplegics with partial diaphragm function from the ventilator, analogous to progressive resistive exercise training. In the three patients presented we were successful in discontinuing mechanical ventilation without subsequent decompensation requiring re-intubation.

\section{References}

Bellemare F, Grassino A 1982 Evaluation of human diaphragm fatigue. Fournal of Applied Physiology 53(5):1196-1206.

COHEN CA, ZAGELBAUM G 1982 Clinical manifestations of inspiratory muscle fatigue. The American fournal of Medicine 73:308-316.

FUFL-MEYER DR 1972 A model for treatment of impaired ventilatory function in tetraplegia patients. Scandinavian fournal of Rehabilitation Medicine 3:168-177.

Gross D, LADD HEW 1980 The effect of training on strength and endurance of the diaphragm in quadriplegia. The American fournal of Medicine 68:27-35.

HaAs Pineda, HaAs Axen 1979 Pulmonary Therapy and Rehabilitation. The Williams and Wilkins Company.

LIEBERMAN DA, FAULKNeR JD 1973 Performance and histochemical composition of guinea pig and human diaphragm. Fournal of Applied Physiology 34:233-237.

LEITH DE, BRADLEY M (1976) Ventilatory muscle strength and endurance training. fournal of Applied Physiology 41(4):508-516.

McMichan JC, Michel L, Westbrook PR (1980) Pulmonary dysfunction following traumatic quadriplegia. $\mathcal{F} A M A 243(6): 528-531$.

Roussos CS, MACKLEM PT 1977 Diaphragmatic fatigue in man. Fournal of Applied Physiology 43(2):189-197.

SHAFFER TD, WOLFSON MR, BHUTANI VK 1981 Respiratory muscle function, assessment and training. PT 61:1711-1723. 